\title{
Hydrophobic Ultrafine Hyaluronic Acid Nanofibers
}

\author{
Marketa Klicova $^{1}$, Andrea Klapstova ${ }^{1}$, Lukas Volesky ${ }^{2}$, Vaclav Liska ${ }^{3}$, Jachym Rosendorf ${ }^{3}$, Richard \\ Palek $^{3}$ and Jana Horakova ${ }^{1}$ \\ ${ }^{1}$ Technical University of Liberec, Faculty of Textile Engineering, Department of Nonwovens and Nanofibrous Materials \\ Studentska 2, 46117 Liberec, Czech Republic \\ marketa.klicova@tul.cz \\ ${ }^{2}$ Technical University of Liberec, Faculty of Mechanical Engineering, Department of Material Science \\ Studentska 2, 46117 Liberec, Czech Republic \\ lukas.volesky@tul.cz \\ ${ }^{3}$ Charles University, Faculty of Medicine in Pilsen, Biomedical Center \\ alej Svobody 1655/76, 32300 Plzen, Czech Republic \\ liskav@fnplzen.cz; jachymrosendorf@gmail.com; palekrichard@gmail.com
}

\section{Extended Abstract}

Hyaluronic acid (HA) is a naturally occuring biopolymer, which exhibits biocompatibility, ability to form hydrogels, has viscoelastic properties and is biodegradable. Thus HA is broadly used in applications based on healthcare, dermatology, pharmacy and tissue engineering [1], [2]. In recent years there has been a growing interest in preparing nanofibers based on HA as these fibers can act like a scaffold for regenerative medicine. Nanofibers are widely prepared by electrospinning, since it provides reproducible method for creating the uniform nanofibrous mats. However, the electrospinning of large molecular weight biopolymers such as HA is difficult due to its polyelectrolytic nature and high viscosity in aqueous solutions [3]. Also, the stability of HA nanofibrous mats in vitro or in vivo is limited, as HA dissolves immediately after contact with water. On the other hand, with commonly used techniques for crosslinking of polymers it is still difficult to maintain biocompatibility.

This paper introduces the conditions and methods for preparation of biocompatible, biodegradable, stable and uniform HA nanofibrous mats. In this study, the conditions for easy reproducible electrospinning of high molecular weight (1,2 MDa) $\mathrm{HA}$ in concentration $0,5 \% \mathrm{w} / \mathrm{w}$ in aqueous solutions were found. It was proven there is no need to add toxic solvents or blend HA with other polymers for enhancing the electrospinnability and that contrasts with other studies such as [3]-[5]. The ultrafine fibers of HA were prepared via needle-less method called Nanospider ${ }^{\mathrm{TM}}$. The morphology was carried out by the scanning electron microscope (SEM) and it was found that the bead free fibers with an average diameter of $(79,4 \pm 18,6) \mathrm{nm}$ were prepared.

To increase the stability in aqueous solutions the plasma modification method was chosen. The surface treatment was performed by cold methane plasma, RF Frequency Plasma Assisted Chemical Vapor Deposition (PACVD) method. This technique is a chemical deposition method, chemical reactions were conducted in a high-frequency electric field in a vacuum chamber into which methane gas was injected. Other parameters included vacuum pressure [Pa], voltage [V], gas flow [sccm] and time [min]. In all processes, the parameters were kept constant at the following values: $\mathrm{p}=20 \mathrm{~Pa} ; \mathrm{U}=200 \mathrm{~V} ; \mathrm{CH}_{4}=20$ $\mathrm{sccm} ; \mathrm{t}=3 \mathrm{~min}$. The SEM observation show that the diameter of nanofibers was increased to $(113,77 \pm 30,84) \mathrm{nm}$. The important result is, that the hydrophobicity of the HA nanofibers was rapidly increased. Accroding to contact angle measurement via sessile drop technique, the contact angle was increased from $(46,59 \pm 15,19)^{\circ}$ to $(106,29 \pm 10,23)^{\circ}$. The FTIR spectra showed no difference before and after treatment and it means, that the chemical structure of HA remained unchanged after plasma exposure. The stability of HA layer was mainted during in vitro testing and no change in morphological structure was observed. The cytotoxicity of the HA nanofibrous mat after plasma treatment was assesed via in vitro testing accroding to ISO 10993-5:2009 Biological evaluation of medical devices - Part 5: Tests for in vitro cytotoxicity. It was found, that the treated HA nanofibers are cytocompatible and even promote cell adhesion and proliferation of mouse $3 \mathrm{~T} 3$ fibroblasts. 


\section{Acknowledgements}

This work was supported by Student's grant competition (SGS) 2019 at the Technical University of Liberec.

\section{References}

[1] H. S. Yoo, E. A. Lee, J. J. Yoon, and T. G. Park, "Hyaluronic acid modified biodegradable scaffolds for cartilage tissue engineering," Biomaterials, vol. 26, no. 14, pp. 1925-1933, May 2005.

[2] C. G. Boeriu, J. Springer, F. K. Kooy, L. A. M. van den Broek, and G. Eggink, "Production Methods for Hyaluronan," Int. J. Carbohydr. Chem., vol. 2013, p. e624967, Mar. 2013.

[3] E. K. Brenner, J. D. Schiffman, E. A. Thompson, L. J. Toth, and C. L. Schauer, "Electrospinning of hyaluronic acid nanofibers from aqueous ammonium solutions," Carbohydr. Polym., vol. 87, no. 1, pp. 926-929, Jan. 2012.

[4] Y. Ji et al., "Electrospun three-dimensional hyaluronic acid nanofibrous scaffolds," Biomaterials, vol. 27, no. 20, pp. 3782-3792, Jul. 2006.

[5] Y. Liu, G. Ma, D. Fang, J. Xu, H. Zhang, and J. Nie, "Effects of solution properties and electric field on the electrospinning of hyaluronic acid," Carbohydr. Polym., vol. 83, no. 2, pp. 1011-1015, Jan. 2011. 